\title{
Microgravity protein crystal growth for X-ray and neutron crystallography: What works and what would not!
}

\author{
Joseph D. Ng ${ }^{1,2}$, James K. Baird ${ }^{1}$ and Noriko Inoguchi ${ }^{1,2}$
}

${ }^{1}$ Department of Biological Sciences, University of Alabama in Huntsville, Huntsville, AL 35899, USA; ${ }^{2} \mathrm{iXpressGenes} \mathrm{Inc.,} \mathrm{Hudson} \mathrm{Alpha} \mathrm{Institute} \mathrm{for} \mathrm{Biotechnology,} 601$ Genome Way, Huntsville, AL 35806, USA

Macromolecular crystallization experiments in microgravity have been conducted for over three decades aboard the Space Shuttle and in the International Space Station. The primary motivation to study crystal growth in space is based on the concept that a microgravity environment influences macromolecular crystal growth by minimizing buoyant convection flows, limiting nucleation sites, and eliminating crystal sedimentation. Although the effectiveness of obtaining improved protein crystals in space has not gain substantial recognition, there is much evidence that space-grown protein crystals diffract X-rays to higher resolution with improved diffraction intensity compared to those are Earth-grown. Moreover, protein crystals that have been successfully grown in space are usually bigger in volume compared to their Earth counterparts. While obtaining large protein crystals is not as imperative as it was in the past for X-ray crystallography, it is absolutely still necessary for Neutron Macromolecular Crystallography (NMC). This talk will provide a brief historical perspective of protein crystal growth in space, discuss what has and has not worked and predict what proteins may be most suitable for crystallization under microgravity. Results from recent crystallization experiments on the ISS will be shown and its application towards NMC and drug discovery will be discussed. 